\title{
Intravitreous anti-VEGF for diabetic retinopathy: hopes and fears for a new therapeutic strategy
}

\author{
R. Simó • C. Hernández
}

Received: 8 December 2007 / Accepted: 21 February 2008 /Published online: 11 April 2008

(C) Springer-Verlag 2008

\begin{abstract}
Vascular endothelial growth factor (VEGF) plays a key role in the development of both proliferative diabetic retinopathy (PDR) and diabetic macular oedema (DMO). In recent years, anti-VEGF agents have emerged as new approaches to the treatment of these devastating diabetic complications. Although Phase III studies in the diabetic population are needed, intravitreal anti-VEGF therapy is currently being used in clinical practice. Intravitreal injection is an effective means of delivering anti-VEGF drugs to the retina. However, this is an invasive procedure associated with potentially serious complications, such as endophthalmitis or retinal detachment, which may be significant for patients requiring serial treatment over many years. In addition, although delivered within the vitreous, anti-VEGF drugs could pass into the systemic circulation, which could potentially result in hypertension, proteinuria, increased cardiovascular events and impaired wound healing. Pegaptanib, ranibizumab and bevacizumab are the currently available anti-VEGF agents. Ranibizumab and bevacizumab block all VEGF isoforms, thus impairing both physiological and pathological neovascularisation. Pegaptanib only blocks the $\mathrm{VEGF}_{165}$ isoform, and would therefore seem the best option for avoiding systemic adverse effects in diabetic
\end{abstract}

\section{R. Simó • C. Hernández}

Centro de Investigación Biomédica en Red de Diabetes y

Enfermedades Metabólicas (CIBERDEM.

Carlos III Health Institute) and Diabetes and Metabolism Research

Unit, Institut de Recerca Hospital Universitari Vall d'Hebron,

Universitat Autònoma de Barcelona,

Barcelona, Spain

\section{R. Simó $(\bowtie)$}

Diabetes and Metabolism Research Unit,

Institut de Recerca Hospital Universitari Vall d'Hebron,

Universitat Autònoma de Barcelona,

Pg Vall d'Hebron 119-129,

08035 Barcelona, Spain

e-mail: rsimo@ir.vhebron.net patients, although this remains to be demonstrated in clinical trials. In this regard, head-to-head studies designed to evaluate not only the efficacy, but also the systemic adverse effects of these drugs in a high-risk population such as diabetic patients are warranted.

Keywords Bevacizumab - Diabetic retinopathy Pegaptanib · Ranibizumab · VEGF inhibitors .

VEGF isoforms
Abbreviations
AMD age-related macular degeneration
CNV choroidal neovascularisation
DMO diabetic macular oedema
DR diabetic retinopathy
$\mathrm{FAb}$ fragment antigen binding
FDA Food and Drug Administration
$\mathrm{mAb}$ monoclonal antibody
PDR proliferative diabetic retinopathy
VEGF vascular endothelial growth factor

\section{Introduction}

Proliferative diabetic retinopathy (PDR) is a leading cause of blindness and visual impairment among adults aged $<40$ years in the developed world [1]. Diabetic macular oedema (DMO), another important event that occurs in the setting of diabetic retinopathy (DR), is more frequent in type 2 than type 1 diabetes [2]. Although DMO does not cause total blindness, it frequently leads to a severe loss of central vision. Because of the high prevalence of type 2 diabetes, DMO is the main cause of visual impairment in diabetic patients. In addition, DMO is almost invariably present when PDR is detected in type 2 diabetes [3]. 
Our understanding of the pathophysiology of both PDR and DMO is increasing as new biochemical pathways are identified. Hypoxia-driven angiogenesis is a crucial pathway in the development of PDR, whereas the leakage of plasma from the small blood vessels in the macula following the disruption of the tight junctions of the blood-retinal barrier is the main factor responsible for DMO. Vascular endothelial growth factor (VEGF) plays an essential role in the development of both PDR and DMO [4]. In this regard, it is significant that anti-VEGF strategies involving intravitreal injections have recently emerged as potential new therapies for PDR [5-12] and DMO [13-20]. The use of intravitreous anti-VEGF treatments in DR has yet to be evaluated in Phase III trials. Nevertheless, because of the spectacular results reported in neovascular age-related macular degeneration (AMD) [21-24], this treatment is currently used by many ophthalmologists in PDR and DMO. Given that antiVEGF drugs delivered within the vitreous could pass into the systemic circulation, VEGF blocking is likely to produce systemic adverse effects, which could potentially be serious in diabetic patients. For this reason, and because of the increasing off-label use of anti-VEGF agents, the clinician has to know not only the potential advantages of, but also the concerns surrounding these emerging treatments for DR.

In this paper we discuss the advantages and disadvantages of anti-VEGF treatment from a physiopathological standpoint, to place physicians who treat diabetic patients in a better position to decide whether or not to recommend this type of treatment.

\section{VEGF isoforms and neovascularisation}

By alternative exon splicing of a single gene consisting of eight exons, several VEGF isoforms can be generated [25]. There are short isoforms such as $\mathrm{VEGF}_{121}$, which is freely diffusible, and larger isoforms such as $\mathrm{VEGF}_{189}$ and $\mathrm{VEGF}_{206}$, which contain the heparin-binding domain and remain as non-diffusible extracellular matrix-bound forms. VEGF $_{165}$ possesses a combination of these properties, and although it is diffusible, a significant fraction remains bound to the cell surface and the extracellular matrix.

All VEGF isoforms except VEGF $_{121}$ contain a plasmin cleavage site and may theoretically be cleaved by plasmin to generate the smaller, diffusible $\mathrm{VEGF}_{110}$ isoform [26]. VEGF $_{110}$ can stimulate endothelial cell growth and induce vascular permeability; however, it is not as mitogenically potent as $\mathrm{VEGF}_{165}$.

VEGF $_{165}$ is the most abundantly expressed VEGF isoform, and has the optimal characteristics of bioavailability combined with high biological potency. Using a mouse model of ocular neovascularisation, Ishida et al. [27] showed that both the absolute and relative levels of
$\mathrm{VEGF}_{164}$ (the equivalent of $\mathrm{VEGF}_{165}$ in humans) increased to a greater degree during pathological neovascularisation than during physiological neovascularisation. Intravitreous injection of pegaptanib (a selective blocker of $\mathrm{VEGF}_{164}$ ) potently suppressed the pathological neovascularisation, whereas it had little or no effect on physiological neovascularisation. In parallel experiments, genetically altered VEGF $_{164}$-deficient mice exhibited no difference in physiological neovascularisation compared with wild-type $(\mathrm{VEGF}+/+)$ controls. In contrast, administration of a VEGF receptor $1-\mathrm{Fc}$ fusion protein $(1 \mu \mathrm{g})$, which blocks all VEGF isoforms, led to significant suppression of both pathological and physiological neovascularisation. Therefore, at least in experimental retinopathy, $\mathrm{VEGF}_{164}$ plays an essential role in pathological, but not in physiological, neovascularisation, or in other words, VEGF isoforms other than $\mathrm{VEGF}_{164}$, in combination, may be sufficient to promote normal physiological neovascularisation. These findings have led to $\mathrm{VEGF}_{164 / 165}$ being proposed as an optimal target for blockade.

\section{Physiological actions of VEGF}

VEGF is crucial for embryonic and early postnatal vasculogenesis and angiogenesis $[25,26]$. In adults, VEGF acts at several levels in the vessel beds: it is a survival factor for endothelial cells, it increases microvascular permeability and is a potent vasodilator [25]. In the kidney, glomerulogenesis and renal glomerular capillary function are under the strict gene-dose-dependent control of VEGF [28]. VEGF also participates in skeletal muscle regeneration, cardiac remodelling and endochondral bone formation. It also plays an important role in the female reproductive cycle $[25,26]$.

Apart from physiological actions, VEGF has other effects that, although triggered by pathological stimuli, are very desirable. These include the capacity to promote the formation of collateral vessels, which is essential for recovery following ischaemic events [25], and a substantial role in wound healing [29]. In this regard it is important to note that VEGF acts as a chemoattractant to mobilise endothelial cells from the bone marrow [30].

\section{VEGF inhibitors}

Pegaptanib (Macugen, Eyetech Pharmaceuticals/Pfizer) is a PEGylated (i.e. conjugated to polyethylene glycol) neutralising RNA aptamer with an extremely high affinity for human VEGF $_{165}$ [31]. Aptamers are chemically synthesised and modified oligonucleotides composed of single-stranded nucleic acids that adopt a specific three-dimensional conformation, allowing them to bind with high specificity and 
affinity to molecular targets in a manner similar to that of monoclonal antibodies [32]. An important feature of aptamers is that, unlike a number of recombinant human proteins, they are essentially non-immunogenic. Intravitreal administration of pegaptanib has been shown to significantly inhibit leucostasis, pathological retinal neovascularisation and VEGF-mediated vascular leakage in rodents [33, 34]. Pegaptanib was approved by the US Food and Drug Administration (FDA) for the treatment of exudative AMD in December 2004.

Ranibizumab (Lucentis, Genentech/Roche) was designed to specifically treat neovascular AMD by manipulating the structure of a murine full-length monoclonal antibody ( $\mathrm{mAb}$ ) A.4.6.1 directed against human VEGF-A [35]. The fragment antigen binding (FAb) fragment of A.4.6.1 was humanised and referred to as rhuFab V1 (Fab-12). It was then affinitymatured using phage display technology to generate the Y0317 variant [36], also known as ranibizumab (rhuFab $\mathrm{V} 2$ ). The FAb form was developed after studies in rhesus monkeys reported that intravitreal injection of a full-length antibody (trastuzumab [Herceptin, Roche]) did not produce full retinal penetration [37]. In contrast to pegaptanib, ranibizumab binds to and inhibits the biological activity of all isoforms of human VEGF. In non-human primate studies of laser-induced choroidal neovascularisation (CNV), intravitreal injection of ranibizumab reduced the incidence of new vessel formation and decreased leakage from established vessels [38]. The FDA approved ranibizumab for wet AMD in June 2006.

Bevacizumab (Avastin, Genentech/Roche) was produced by humanisation of the mouse anti-VEGF Mab A.4.6.1 and, like ranibizumab, binds all isoforms of VEGF. However, whereas ranibizumab is a $48 \mathrm{KDa} \mathrm{FAb}$ fragment, bevacizumab is a complete $149 \mathrm{KDa}$ antibody and therefore possesses two antigen-binding domains [39]. The different molecular masses of the two drugs could result in differences with respect to ability to reach the site of action (smaller may be better) and to stay in the eye after injection (larger may be better) [37]. Bevacizumab was originally developed for systemic treatment of colon cancer and was approved by the FDA in February 2004 for the treatment of metastatic colorectal cancer. Despite the lack of any randomised trial data, intravitreal injection of bevacizumab has become a popular off-label treatment for neovascular AMD, mainly because it is perceived to be as effective as ranibizumab but is much cheaper [40].

\section{Potential adverse effects of VEGF blockade}

Systemic adverse effects Although the anti-VEGF drugs are administered by injection through the sclera into the vitreous cavity, entry into the circulation occurs, which could potentially cause systemic adverse effects. Because anti-VEGF treatment for PDR or DMO is potentially required for years, chronic VEGF inhibition may cause adverse effects that are not immediately apparent.

The potential consequences of VEGF blockade are displayed in the text box

\begin{tabular}{|l|}
\hline $\begin{array}{l}\text { Potential adverse effects of blocking VEGF } \\
\text { - Hypertension }{ }^{\mathrm{a}} \\
\text { - Proteinuria }{ }^{\mathrm{a}}\end{array}$ \\
- Impairment of wound healing \\
development \\
- Inhibition of bone growth \\
- Infertility \\
- Inhibition of skeletal muscle \\
regeneration and cardiac remodelling \\
$\begin{array}{l}\text { worrying in } \\
\text { the diabetic } \\
\text { population }\end{array}$ \\
${ }^{\text {S Surrogate markers of systemic VEGF inhibition }}$ \\
\hline
\end{tabular}

Given that VEGF is involved in the control of vascular tone and glomerular capillary function, the main consequences are hypertension and proteinuria. Hypertension is caused by the predominance of vasoconstriction, and proteinuria is secondary to the swelling of the glomerular endothelial cells and the collapse of capillary loops. In fact, both hypertension and proteinuria have been proposed as surrogate markers of systemic VEGF inhibition [41] and have frequently been reported as adverse effects of systemic administration of bevacizumab in clinical trials in oncology [42]. Other potential consequences of blocking VEGF include infertility, inhibition of bone growth, inhibition of skeletal muscle regeneration and cardiac remodelling, impairment of wound healing and impairment of collateral vessel development.

Impaired wound healing might be involved in the increase of bleeding associated with systemic anti-VEGF therapy [43]. In addition, the impairment of collateral vessel development might be involved in cardiovascular ischaemic events reported in trials using anti-VEGF treatment. In this regard, it is important to note the differential regulation of VEGF and its receptors between microvascular and cardiac tissues in diabetic patients. High levels of VEGF and its receptors are observed in DR and diabetic nephropathy, and this is particularly crucial for the development of DR. In contrast, low levels of VEGF and its receptors are found in the myocardium of diabetic patients [44], resulting in inadequate collateral formation. These findings strongly suggest that diabetic patients might be more prone to the development of myocardial ischaemia if circulating VEGF is blocked. 
Diabetes is an extremely important risk factor for cardiovascular disease, and cardiovascular disease is the main cause of mortality in diabetes $[45,46]$. Diabetic nephropathy is the leading cause of end-stage renal disease [47]. Moreover, the impaired wound healing is involved in the development of foot ulcers that account for up to $50 \%$ of non-traumatic leg amputations [48-50]. Thus, the potential consequences of blocking VEGF (i.e. hypertension, proteinuria and impaired wound healing and collateral vessel development) are particularly worrying in the setting of diabetes.

Ocular adverse effects Endophthalmitis, traumatic injury of the lens, and retinal detachment are the most frequent ocularrelated adverse effects, but in all cases the rate is less than $1.5 \%$ per patient per year [21-24]. While the incidence of serious complications associated with intraocular injections is low, cumulative risk exposure may be significant for patients, such as those with diabetes, who require serial treatments over many years.

Apart from the side effects of the intravitreous injection itself, there are other potential intraocular adverse effects that arise as a consequence of VEGF blockade. Secretion of VEGF by retinal pigment epithelium helps to maintain the choriocapillaris (the vascular network that underlies the retina) [51, 52] and has a neuroprotective effect in the ischaemic retina [45]. In this regard, a dose-dependent decrease in ganglion cells has been reported following injection of an antibody that blocks all VEGF isoforms in rats [53]. Furthermore, this loss of neural cells was apparent prior to any observable effect on the vasculature. Interestingly, when the effects of VEGF were blocked by pegaptanib, which does not bind to $\mathrm{VEGF}_{120}$ (the equivalent of $\mathrm{VEGF}_{121}$ in humans), there was no decrease in the number of retinal ganglion cells. Other authors have not found the intravitreous injection of bevacizumab (which blocks all circulating VEGF isoforms) to have any direct toxic effects on retinal ganglion cells [54, 55]. Moreover, bevacizumab seems to have neutral effects on several types of retinal cell (including ganglion cells) in culture [56-58]. However, it should be noted that although no evidence of retinal toxicity can be observed by light microscopy, mitochondrial disruption in the inner segments of photoreceptors (detected by electron microscopy) and a high rate of apoptosis have been reported in rabbit eyes following the intravitreal administration of bevacizumab [59]. In addition, intravitreal bevacizumab significantly reduces choriocapillaris endothelial cell fenestration in primate eyes [60]. These findings could have clinical implications, since the long-term neutralisation of retinal VEGF may have unintended consequences, including loss of neural retina cells and increased risk of circulation disturbances in the choriocapillaris [61]. The development of a VEGF inhibitor that blocks the pathological effects of VEGF while sparing its neuroprotective effects continues to be an important aim of drug developers For these, reasons, postmarketing surveillance to determine the long-term safety of these drugs is clearly needed.

\section{Results of clinical trials using anti-VEGF drugs}

Intravenous administration Intravenous perfusion of bevacizumab has been used as an adjuvant treatment for patients with metastatic breast, colorectal or renal cancer $[62,63]$.

There has been only one study of intravenous bevacizumab in eye diseases; specifically, the treatment of 18 patients with neovascular AMD [64]. In this uncontrolled study a dose of $5 \mathrm{mg} / \mathrm{kg}$ was used, and one or two additional doses were given at 2 week intervals. At 24 weeks of follow-up the visual acuity score had increased by 14 letters and retinal thickness had decreased significantly. Regarding systemic side effects, only a mild and transient increase in blood pressure was reported. Despite these impressive results, it should be noted that this study was not specifically designed to detect adverse effects. In addition, patients with cardiovascular disease, proteinuria or those receiving anticoagulation treatment were excluded. Therefore, the low rate of adverse effects cannot be applied to the real world population, let alone to diabetic patients. However, because of its effectiveness and practical feasibility, intravenous antiVEGF therapy, using selective anti-VEGF 165 agents (i.e. pegaptanib) in particular, could merit study in a large clinical trial.

Intravitreous administration The main prospective clinical trials have been conducted in patients with AMD using pegaptanib and ranibizumab [21-24]. Table 1 displays the efficacy of the agents tested and the rates of adverse effects detected in these studies. Pegaptanib seems to be somewhat less effective than ranibizumab, but appears to be associated with fewer adverse effects. The three pivotal studies on ranibizumab reported an increased frequency of cardiovascular events (including stroke) and bleeding relative to the placebo group, although these increases were not statistically significant.

In spite of these positive results there are two main concerns that should be addressed. First, because these trials were not designed to detect small differences in risk, much larger cohorts would be necessary to allow the evaluation of systemic adverse effects. For example, analysis of the results of the Minimally Classic/Occult Trial of the Anti-VEGF Antibody Ranibizumab in the Treatment of Neovascular Age-Related Macular Degeneration (MARINA) and AntiVEGF Antibody for the Treatment of Predominantly Classic Choroidal Neovascularization in Age-Related Macular Degeneration (ANCHOR) studies together reveals a significantly higher rate of bleeding in the group of treated patients. 
Table 1 Efficacy and systemic adverse effects observed in the main prospective studies on anti-VEGF drugs

\begin{tabular}{|c|c|c|c|c|c|c|c|c|}
\hline Drug & $\begin{array}{l}\text { Clinical trial } \\
\text { [Ref.] }\end{array}$ & $N /$ duration & $\begin{array}{l}\text { Dose } \\
(\mathrm{mg})\end{array}$ & $\begin{array}{l}\text { Efficacy }^{a} \\
(\%)\end{array}$ & $\begin{array}{l}\text { Cardiovascular } \\
\text { events }^{\mathrm{b}}(\%)\end{array}$ & $\begin{array}{l}\text { Hypertension } \\
(\%)\end{array}$ & $\begin{array}{l}\text { Proteinuria } \\
(\%)\end{array}$ & $\begin{array}{l}\text { Bleeding } \\
(\%)\end{array}$ \\
\hline Pegaptanib & VISION [21] & $1,186 / 1$ year & $0.3,1.3$ & 70 vs 55 & 6 vs 6 & 10 vs 10 & - & 2 vs 3 \\
\hline Ranibizumab & MARINA [22] & $716 / 2$ year & $0.3,0.5$ & 92 vs 53 & 4.6 vs 3.8 & 17 vs 16 & No increase & 9 vs 5 \\
\hline Ranibizumab & ANCHOR [23] & $423 / 1$ year & $0.3,0.5$ & 96.4 vs 64.3 & 4.3 vs 2.1 & 4 vs 8 & No increase & 6 vs 2 \\
\hline Ranibizumab & FOCUS [24] & $162 / 1$ year & 0.5 & 90.5 vs 68 & 8.6 vs 5.4 & - & - & - \\
\hline
\end{tabular}

There were no statistical differences regarding systemic adverse effects between patients who received anti-VEGF agents and placebo

${ }^{a}$ Primary endpoint of proportion of participants losing fewer than 15 letters from baseline visual acuity (treatment vs sham all $p<0.0001$ )

${ }^{\mathrm{b}}$ Including stroke

ANCHOR, Anti-VEGF Antibody for the Treatment of Predominantly Classic Choroidal Neovascularization in Age-Related Macular Degeneration; FOCUS, Rhufab V2 Ocular Treatment Combining the Use of Visudine to Evaluate Safety; MARINA, Minimally Classic/Occult Trial of the Anti-VEGF Antibody Ranibizumab in the Treatment of Neovascular Age-Related Macular Degeneration; VISION, VEGF Inhibition Study in Ocular Neovascularization

Second, the overall mortality rates were low given the advanced mean age of these populations (nearly 80 years), and this could be mainly due to the exclusion of patients with a history of, or risk factors for cardiovascular disease.

Several studies have reported very promising results in patients with diabetes. In a prospective, double-blind, multicentre, dose-ranging, controlled trial that included 172 patients with DMO, participants assigned to pegaptanib had better visual acuity outcomes, were more likely to show a reduction in central retinal thickness, and were deemed less likely to need additional therapy with photocoagulation at follow-up (36 weeks) [13]. In addition, most of the participants with retinal neovascularisation at baseline who were assigned to pegaptanib showed a regression of neovascularisation by week 36 [65]. Uncontrolled studies using ranibizumab and bevacizumab have also found a rapid regression of retinal neovascularisation, an improvement in visual acuity and a decrease in retinal thickness, even in non-responders to conventional treatment [5-20]. Moreover, bevacizumab is currently used by many ophthalmologists as a pretreatment of vitrectomy for severe PDR. Nevertheless, larger studies investigating not only the effectiveness, but also the systemic adverse effects of these agents in the diabetic population are still needed.

\section{Concluding remarks}

Intravitreal injection is used for effective delivery of antiVEGF drugs to the retina. The results obtained in AMD have been very impressive, and the preliminary data in DR are promising. However, this is an invasive procedure, with the potential for blinding sequelae such as endophthalmitis and retinal detachment. In addition, although delivered within the vitreous, anti-VEGF drugs could pass into the systemic circulation. Hypertension, proteinuria and increased cardio- vascular events and bleeding associated with impaired wound healing are the potential consequences of the systemic inhibition of VEGF, and will be particularly worrisome in the diabetic population. Nevertheless, in patients with AMD, intravitreous injection of anti-VEGF agents does not produce a significantly higher frequency of adverse effects compared with placebo. However, trial data on the safety of anti-VEGF treatments in AMD patients might not be generalisable to the wider community, and even less so to diabetic patients, and controlled trials specifically designed to evaluate the long-term systemic adverse effects of anti-VEGF therapy are lacking.

Pegaptanib, ranibizumab and bevacizumab are the currently available anti-VEGF agents. Bevacizumab has not been approved for intravitreous delivery. However, it is often used off-label by many ophthalmologists because it appears to be as effective as pegaptanib or ranibizumab but is much cheaper. Ranibizumab and bevacizumab block all circulating VEGF isoforms $\left(\mathrm{VEGF}_{165}, \mathrm{VEGF}_{121}\right.$ and $\mathrm{VEGF}_{110}$ ), thus impairing both physiological and pathological neovascularisation. By contrast, $\mathrm{VEGF}_{165}$ is selectively targeted by pegaptanib. Given that $\mathrm{VEGF}_{165}$ plays an essential role in pathological but not in physiological neovascularisation, pegaptanib seems the best option for avoiding systemic adverse effects in diabetic patients. However, this theoretical advantage remains to be demonstrated in clinical trials. In this regard, head-to-head studies of these drugs in the diabetic population would probably provide the most useful information.

Diabetes is a major cardiovascular risk factor and is associated with other risk factors for cardiovascular disease. Consequently, any chronic therapies that could further increase this risk have important public health implications. Therefore, more clinical trials designed to evaluate not only efficacy, but also systemic adverse effects are needed before launching anti-VEGF therapy in a high-risk population such as diabetic patients. 
Acknowledgements This study was supported by grants from Instituto Carlos III de Salud (CIBERDEM; Carlos III Health Institute), Ministerio de Ciencia y Tecnología (Spanish Ministry of Science and Technology; SAF2006-05284) and Acadèmia de Ciències Mèdiques de Catalunya i Balears (ACMCB; Medical Sciences of Catalonia and Balearics).

Duality of interest The authors declare that there is no duality of interest associated with this manuscript.

\section{References}

1. Congdom N, Friedman DS, Lietman T (2003) Important causes of visual impairment in the world today. JAMA 290:2057-2060

2. Tong L, Vernon SA, Kiel W, Sung V, Orr GM (2001) Association of macular involvement with proliferative retinopathy in type 2 diabetes. Diabet Med 18:388-394

3. Klein R, Klein BE, Moss SE, Davis MD, Demets DL (1984) The Wisconsin Epidemiologic Study of Diabetic Retinopathy. III. Prevalence and risk of diabetic retinopathy when age at diagnosis is 30 or more years. Arch Ophthalmol 102:527-532

4. Cladwell RB, Bartoli M, Behzadian MA et al (2003) Vascular endothelial growth factor and diabetic retinopathy: pathophysiological mechanisms and treatment perspectives. Diabetes Metab Res Rev 19:442-455

5. Hernández C, Simó R (2007) Strategies for blocking angiogenesis in diabetic retinopathy: from basic science to clinical practice. Expert Opin Invest Drugs 16:1209-26

6. Spaide RF, Fisher YL (2006) Intravitreal bevacizumab (Avastin) treatment of proliferative diabetic retinopathy complicated by vitreous hemorrhage. Retina 26:275-278

7. Oshima Y, Sakaguchi H, Gomi F, Tano Y (2006) Regression of iris neovascularization after intravitreal injection of bevacizumab in patients with proliferative diabetic retinopathy. Am J Ophthalmol 142:155-158

8. Chen E, Park CH (2006) Use of intravitreal bevacizumab as a preoperative adjunct for tractional retinal detachment repair in severe proliferative diabetic retinopathy. Retina 26:699-700

9. Avery RL, Pearlman J, Pieramici DJ et al (2006) Intravitreal bevacizumab (Avastin) in the treatment of proliferative diabetic retinopathy. Ophthalmology 113:1695.e1-15

10. Jorge R, Costa RA, Calucci D, Cintra LP, Scott IU (2006) Intravitreal bevacizumab (Avastin) for persistent new vessels in diabetic retinopathy (IBEPE study). Retina 26:1006-1013

11. Arevalo JF, Wu L, Sanchez JG et al (2008) Intravitreal bevacizumab (Avastin) for proliferative diabetic retinopathy: 6months follow-up. Eye DOI 10.1038/sj.eye.6702980

12. Ishikawa K, Honda S, Tsukahara Y, Negi A (2008) Preferable use of intravitreal bevacizumab as a pre-treatment of vitrectomy for severe proliferative diabetic retinopathy. Eye DOI 10.1038/sj.eye.6702983

13. Cunningham ET Jr, Adamis AP, Altaweel M et al (2005) A Phase II randomized double-masked trial of pegaptanib, an anti-vascular endothelial growth factor aptamer, for diabetic macular edema. Ophthalmology 112:1747-1757

14. Chun DW, Heier JS, Topping TM, Duker JS, Bankert JM (2006) A pilot study of multiple intravitreal injections of ranibizumab in patients with center-involving clinically significant diabetic macular edema. Ophthalmology 113:1706-1712

15. Nguyen QD, Tatlipinar S, Shah SM et al (2006) Vascular endothelial growth factor is a critical stimulus for diabetic macular edema. Am J Ophthalmol 142:961-969

16. Haritoglou C, Krook D, Neubauer A et al (2006) Intravitreal bevacizumab (Avastin) therapy for persistent diffuse diabetic macular edema. Retina 26:999-1005
17. Arevalo JF, Fromow-Guerra J, Quiroz-Mercado H et al (2007) Primary intravitreal bevacizumab (Avastin) for diabetic macular edema: results from the Pan-American Collaborative Retina Study Group at 6-month follow-up. Ophthalmology 114:743-50

18. Diabetic Retinopathy Clinical Research Network (2007) A Phase II randomized clinical trial of intravitreal bevacizumab for diabetic macular edema. Ophthalmology 114:1860-1867

19. Ahmadieh H, Ramezani A, Shoeibi $N$ et al (2008) Intravitreal bevacizumab with or without triamcinolone for refractory diabetic macular edema; a placebo-controlled, randomized clinical trial. Graefes Arch Clin Exp Ophthalmol 246:483-489

20. Kumar A, Sinha S (2007) Intravitreal bevacizumab (Avastin) treatment of diffuse diabetic macular edema in an Indian population. Indian J Ophthalmol 55:451-455

21. Gragoudas ES, Adamis AP, Cunningham ET, Feinsod M, Guyer DR (2004) Pegaptamib for neovascular age-related macular degeneration. N Engl J Med 351:2805-2816

22. Rosenfeld PJ, Brown DM, Heier JS et al (2006) Ranibizumab for neovascular age-related macular degeneration. $\mathrm{N}$ Engl J Med 355:1419-1431

23. Brown DM, Kaiser PK, Michels M et al (2006) Ranibizumab versus verteporfin for neovascular age-related macular degeneration. N Engl J Med 355:1432-1444

24. Heier JS, Boyer DS, Ciulla TA et al (2006) Ranibizumab combined with verteporfin photodynamic therapy in neovascular age-related macular degeneration: year 1 results of the FOCUS Study. Arch Ophthalmol 124:1532-1542

25. Ferrara N (2001) Role of vascular endothelial growth factor in regulation of physiological angiogenesis. Am J Physiol Cell Physiol 280:C1358-C1366

26. Takahashi H, Shibuya M (2005) The vascular endothelial growth factor (VEGF)/VEGF receptor system and its role under physiological and pathological conditions. Clin Sci (Lond) 109:227-241

27. Ishida $\mathrm{S}$, Usui $\mathrm{T}$, Yamashiro $\mathrm{K}$ et al (2003) $\mathrm{VEGF}_{164}$-mediated inflammation is required for pathological, but not physiological, ischemia-induced retinal neovascularization. J Exp Med 198:483489

28. Eremina V, Sood M, Haigh J et al (2003) Glomerular-specific alterations of VEGF-A expression lead to distinct congenital and acquired renal diseases. J Clin Invest 11:707-716

29. Nissen NN, Polverini PJ, Koch AE, Volin MV, Gamelli RL, DiPietro LA (1998) Vascular endothelial growth factor mediates angiogenic activity during the proliferative phase of wound healing. Am J Pathol 152:1445-1452

30. Asahara T, Takahashi T, Masuda H et al (1999) VEGF contributes to postnatal neovascularization by mobilizing bone marrowderived endothelial progenitor cells. EMBO J 18:3964-3972

31. Yasukawa T, Ogura Y, Tabata Y, Kimura H, Wiedemann P, Honda Y (2004) Drug delivery systems for vitreoretinal diseases. Prog Retin Eye Res 23:253-281

32. White RR, Sullenger BA, Rusconi CP (2000) Developing aptamers into therapeutics. J Clin Invest 106:929-934

33. Eyetech Study Group (2002) Preclinical and Phase 1A clinical evaluation of an anti-VEGF pegylated aptamer (EYE001) for the treatment of exudative age-related macular degeneration. Retina $22: 143-152$

34. Ishida $\mathrm{S}$, Usui $\mathrm{T}$, Yamashiro $\mathrm{K}$ et al (2004) $\mathrm{VEGF}_{164(165)}$ as the pathological isoform: differential leukocyte and endothelial responses through VEGFR1 and VEGFR2. Invest Ophthalmol Vis Sci 45:368-374

35. Kim KJ, Li B, Houck K et al (1992) The vascular endothelial growth factor proteins: identification of biologically relevant regions by neutralizing monoclonal antibodies. Growth Factors 7:53-64 
36. Chen Y, Wiesmann C, Fuh G et al (1999) Selection and analysis of an optimized anti-VEGF antibody: crystal structure of an affinitymatured Fab in complex with antigen. J. Mol Biol 293:865-881

37. Mordenti J, Cuthbertson RA, Ferrara N et al (1999) Comparisons of the intraocular tissue distribution, pharmacokinetics, and safety of ${ }^{125}$ I-labeled full-length and Fab antibodies in rhesus monkeys following intravitreal administration. Toxicol Pathol 27:536-544

38. Krzystolilk MG, Afshari MA, Adamis AP et al (2002) Prevention of experimental choroidal neovascularization with intravitreal anti-vascular endothelial growth factor antibody fragment. Arch Ophthalmol 120:338-346

39. Kaiser PK (2006) Antivascular endothelial growth factor agents and their development: therapeutic implications in ocular diseases. Am J Ophthalmol 142:660-668

40. Rosenfeld PJ (2006) Intravitreal Avastin: the low cost alternative to Lucentis? Am J Ophthalmol 142:141-143

41. Luttun A, Carmeliet P (2003) Soluble VEGF receptor Flt1: the elusive preeclampsia factor discovered? J Clin Invest 11:600-602

42. Zhu X, Wu S, Dahut WL, Parikh CR (2007) Risks of proteinuria and hypertension with bevacizumab, an antibody against vascular endothelial growth factor: systematic review and meta-analysis. Am J Kidney Dis 49:186-193

43. Kamba T, McDonald DM (2007) Mechanisms of adverse effects of anti-VEGF therapy for cancer. Br J Cancer 2007 96:1788-1795

44. Chou E, Suzuma I, Kerrie JW et al (2002) Decreased cardiac expression of vascular endothelial growth factor and its receptors in insulin-resistant and diabetic states: a possible explanation for impaired collateral formation in cardiac tissue. Circulation 105: 373-379

45. Beckman JA, Creager MA, Libby P (2002) Diabetes and atherosclerosis: epidemiology, pathophysiology, and management. JAMA 287:2570-2581

46. Hogan P, Dall T, Nikolov P (2003) Economic costs of diabetes in the U.S. in 2002. Diabetes Care 26:917-932

47. Centres for Disease control and Prevention (CDC) (2005) Incidence of end-stage renal disease among persons with diabetes-United States, 1990-2002. MMWR Morb Mortal Wkly Rep 54:1097-1100

48. Most RS, Sinnock P (1983) The epidemiology of lower extremity amputations in diabetic individuals. Diabetes Care 6:87-91

49. Falanga V (2005) Wound healing and its impairment in the diabetic foot. Lancet 366:1736-1743

50. Dalla Paola L, Faglia E (2006) Treatment for diabetic foot ulcer: an overview strategies. Curr Diabetes Rev 2:431-447

51. Blaauwgeers HG, Holtkamp GM, Rutten H et al (1999) Polarized vascular endothelial growth factor secretion by human retinal pigment epithelium and localization of vascular endothelial growth factor receptors on the inner choriocapillaris. Evidence for a trophic paracrine relation. Am J Pathol 155:421-428
52. Marneros AG, Fan J, Yokoyama Y et al (2005) Vascular endothelial growth factor expression in the retinal pigment epithelium is essential for choriocapillaris development and visual function. Am J Pathol 167:1451-1459

53. Nishijima K, Yin-Shan N, Zhong L et al (2007) Vascular endothelial growth factor-A is a survival factor for retinal neurons and a critical neuroprotectant during the adaptive response to ischemic injury. Am J Pathol 171:53-67

54. Bakri SJ, Cameron JD, McCannel CA, Pulido JS, Marler RJ (2006) Absence of histologic retinal toxicity of intravitreal bevacizumab in a rabbit model. Am J Ophthalmol 142:162-164

55. Iriyama A, Chen YN, Tamaki Y, Yanagi Y (2007) Effect of the anti-VEGF antibody on the retinal ganglion cell of rat. $\mathrm{Br} \mathrm{J}$ Ophthalmol 91:1230-1233

56. Luthra S, Narayanan R, Marques LE et al (2006) Evaluation of in vitro effects of bevacizumab (Avastin) on retinal pigment epithelial, neurosensory retinal, and microvascular endothelial cells. Retina 26:512-518

57. Spitzer MS, Wallenfels-Thilo B, Sierra A et al (2006) Antiproliferative and cytotoxic properties of bevacizumab on different ocular cells. Br J Ophthalmol 90:1316-1321

58. Spitzer MS, Yoeruek E, Sierra A et al (2007) Comparative antiproliferative and cytotoxic profile of bevacizumab (Avastin), pegaptanib (Macugen) and ranibizumab (Lucentis) on different ocular cells. Graefes Arch Clin Exp Ophthalmol 245:1837-42

59. Inan UU, Avci B, Kusbeci T, Kaderli B, Avci R, Temel SG (2007) Preclinical safety evaluation of intravitreal injection of full-length humanized vascular endothelial growth factor antibody in rabbit eyes. Invest Ophthalmol Vis Sci 48:1773-1781

60. Peters S, Heiduschka P, Julien S et al (2007) Ultrastructural findings in the primate eye after intravitreal injection of bevacizumab. Am J Ophthalmol 143:995-1002

61. D'Amore PA (2007) Vascular endothelial cell growth factor-a: not just for endothelial cells anymore. Am J Pathol 171:14-18

62. Kabbinavar F, Hurwitz H, Fehrenbacher L et al (2000) Phase II, randomised trial comparing bevacizumab plus fluorouracil (FU)/ lecovorin (LV) with FU/LV alone in patients with metastatic colorectal cancer. N Engl J Med 343:905-914

63. Yang JC, Haworth L, Sherry RM et al (2003) A randomised trial of bevacizumab, an anti-vascular endothelial cell growth factor antibody, for metastatic renal cancer. N Engl J Med 349:427-434

64. Moshfeghi AA, Rosenfeld PJ, Puliafito CA et al (2006) Systemic bevacizumab (Avastin) therapy for neovascular age-related macular degeneration: twenty-four-week results of an uncontrolled open-label clinical study. Ophthalmology 113:2002-2011

65. Adamis AP, Altaweel M, Bressler NM et al (2007) Changes in retinal neovascularization after pegaptanib (Macugen) therapy in diabetic individuals. Ophthalmology 114:615-616 